Three-Dimensional Simulation of Detonation Propagation in a Rectangular Duct by an Improved CE/SE Scheme

This article has been downloaded from IOPscience. Please scroll down to see the full text article.

2011 Chinese Phys. Lett. 28124705

(http://iopscience.iop.org/0256-307X/28/12/124705)

View the table of contents for this issue, or go to the journal homepage for more

Download details:

IP Address: 159.226.231.78

The article was downloaded on 22/03/2012 at 05:59

Please note that terms and conditions apply. 


\title{
Three-Dimensional Simulation of Detonation Propagation in a Rectangular Duct by an Improved CE/SE Scheme*
}

\author{
SHEN Hua(申华) $)^{1,2}$, LIU Kai-Xin(刘凯欣) ${ }^{1,2 * *}$, ZHANG De-Liang(张德良) ${ }^{3}$ \\ ${ }^{1}$ LTCS and College of Engineering, Peking University, Beijing 100871 \\ ${ }^{2}$ Center for Applied Physics and Technology, Peking University, Beijing 100871 \\ ${ }^{3}$ LHD, Institute of Mechanics, Chinese Academy of Sciences, Beijing 100080
}

(Received 25 July 2011)

\begin{abstract}
A genuinely three-dimensional spacetime conservation element and solution element $(\mathrm{CE} / \mathrm{SE})$ scheme is built as simple, consistent and straightforward extensions of an improved high resolution $2 D C E / S E$ scheme. It is applied to examine the mechanism of three-dimensional detonation process in rectangular ducts. The simulations clearly show detailed three-dimensional detonation modes, namely a rectangular mode and a diagonal mode. Furthermore, the formation of unreacted pockets with high density and low temperature behind the detonation is observed for the two modes.
\end{abstract}

DOI:10.1088/0256-307X/28/12/124705

Detonation is still an area of active study due to its practical importance, ${ }^{[1]}$ although it has a long history of research work over 100 years. Previous studies demonstrated that detonation has complicated threedimensional structures. Due to the limitations of experimental equipment and techniques, it is very difficult to capture the complex three-dimensional structure of detonation phenomenon by experiments. Compared with experimental studies, one of the main advantages of numerical simulations is that the local and global profiles of physical variables are available at arbitrary time. Thus numerical simulations act as good complements to experiments for the study of detonation propagation. Most of the previous studies have focused on two-dimensional studies. In recent years, three-dimensional simulations ${ }^{[2-4]}$ have begun to appear as a result of major progress in both numerical methods and available computer facilities. However, most of the three-dimensional simulations suffer from a number of limitations due to the considerable demands on computing resources. Thus, it is necessary to introduce efficient high resolution algorithms to detonation simulations.

The spacetime conservation element and solution element (CE/SE) method, originally proposed by Chang et al.,${ }^{[5,6]}$ is a novel numerical framework for solving hyperbolic conservation laws. It has several attractive features for three-dimensional simulations, especially including: (a) very little dissipation; (b) directional splitting method is not needed for multidimensional cases, resulting in a genuinely multidimensional scheme. Wang et al. ${ }^{[7]}$ tested the accuracy of the $\mathrm{CE} / \mathrm{SE}$ method for computational aeroacoustics problems involving shock waves. The results show that the computational accuracy of the CE/SE method with a first-order Taylor expansion is equivalent to a fourthorder essentially non-oscillatory scheme.

$\mathrm{CE} / \mathrm{SE}$ schemes depend on the definitions of con- servation elements (CE) and solution elements (SE). In Chang's original scheme, the number of CEs is consistent with the number of unknowns designated by the scheme including the mesh variables and their spatial derivatives. Therefore, two CEs are needed at each grid point to derive two discrete equations for $U$ and $U_{x}$ in a one-dimensional case. Similarly, three and four CEs are needed at each grid point in twoand three-dimensional cases, respectively. In order to simplify the construction of the $\mathrm{CE} / \mathrm{SE}$ scheme, a simple type of $\mathrm{CE}$ and $\mathrm{SE}$ on the general quadrilateral mesh was adopted in the two-dimensional scheme by our group. The improved CE/SE scheme is proved to have high accuracy. ${ }^{[8]}$ To date, the improved $\mathrm{CE} / \mathrm{SE}$ method has achieved great success in the simulations of gaseous detonation, ${ }^{[8-10]}$ twophase detonation, ${ }^{[10,11]}$ multi-material elastic-plastic flows. ${ }^{[12-14]}$ and spall fracture. ${ }^{[13,14]}$

In the present study, a genuinely three-dimensional $\mathrm{CE} / \mathrm{SE}$ scheme is built as simple, consistent and straightforward extensions of the improved high resolution $2 \mathrm{D}$ scheme. In addition, the present scheme is implemented on parallel distributed-memory computers using a domain-decomposition approach. It is applied to analyze the mechanism of three-dimensional detonation process in rectangular ducts.

The governing equations used are described by the three-dimensional Euler equations with a source term that represents chemical reactions. In conservation form, these equations may be written in the compact form

$$
\frac{\partial \boldsymbol{U}}{\partial t}+\frac{\partial \boldsymbol{E}}{\partial x}+\frac{\partial \boldsymbol{F}}{\partial y}+\frac{\partial \boldsymbol{G}}{\partial z}=\boldsymbol{S}
$$

where $\boldsymbol{U}=(\rho, \rho u, \rho v, \rho w, e, \rho \alpha, \rho \beta)^{T}, \boldsymbol{E}=\left(\rho u, \rho u^{2}+\right.$ $p, \rho u v, \rho u w,(e+p) u, \rho \alpha u, \rho \beta u)^{T}, \boldsymbol{F}=\left(\rho v, \rho u v, \rho v^{2}+\right.$ $p, \rho v w,(e+p) v, \rho \alpha v, \rho \beta v)^{T}, \boldsymbol{G}=\left(\rho w, \rho u w, \rho v w, \rho w^{2}+\right.$ $p,(e+p) w, \rho \alpha w, \rho \beta w)^{T}, \boldsymbol{S}=\left(0,0,0,0,0, \omega_{\alpha}, \omega_{\beta}\right)^{T}$, in

\footnotetext{
*Supported by the National Natural Science Foundation of China under Grant Nos 10732010 and 10972010.

** Corresponding author. Email: kliu@pku.edu.cn

(c) 2011 Chinese Physical Society and IOP Publishing Ltd
} 
which $\rho, u, v, w$ and $p$ are density, the velocity components of $x$-, $y$ - and $z$-directions and the pressure, respectively. The total energy density $e$ is defined as $e=\frac{p}{\gamma-1}+\frac{\rho\left(u^{2}+v^{2}+w^{2}\right)}{2}+\rho \beta Q$ with $\gamma$ being the specific heat ratio. The two-step reaction model simplifies the complicated chemical reactions to an induction reaction and an exothermic reaction. The progress parameters $\alpha$ and $\beta$, for the induction reaction and the exothermic reaction, respectively, both are zero at first, followed by increase of $\alpha$ to unity, then $\beta$ increases until the equilibrium state is reached. The rates of $\alpha$ and $\beta, \omega_{\alpha}$ and $\omega_{\beta}$, are given as follows: ${ }^{[15]}$

$$
\begin{aligned}
\omega_{\alpha}=\frac{d \alpha}{d t}= & \frac{P}{3 R_{0} T} \exp \left[35.1715-\frac{8530.6}{T}\right. \\
& \left.-7.22 \times 10^{-11} P^{2} \exp \left(\frac{21205}{T}\right)\right],
\end{aligned}
$$

$$
\omega_{\beta}=\frac{d \beta}{d t}=\left\{\begin{array}{ll}
0 & (\alpha<1), \\
(1-\beta) \times a \times \exp \left(-\frac{b}{T}\right)+c & (\alpha=1)
\end{array},\right.
$$

where $a, b$, and $c$ are coefficients, $T$ the temperature and $R_{0}(=8.314 \mathrm{~J} / \mathrm{mol} / \mathrm{K})$ the universal gas constant. The unit of pressure $P$ is atm.

Let $x_{1}=x, x_{2}=y, x_{3}=z$ and $x_{4}=t$ be considered as the coordinates of a four-dimensional Euclidean space $E_{4}$. The decoupling method ${ }^{[9]}$ is applied to treat the source item in Eq. (1). That is to say, the source item is neglected first. Then Eq. (1) can be expressed as $\nabla \cdot \boldsymbol{h}=0$ with $\boldsymbol{h}=(E, F, G, U)$. Gauss' divergence theorem in the spacetime $E_{4}$ implies that Eq. (1) is a differential form of the integral conservation law,

$$
\iiint_{S(V)} \boldsymbol{h} \cdot d \boldsymbol{s}=0
$$

where $S(V)$ is the boundary of an arbitrary spacetime region $V$ in $E_{4}$, and $d \boldsymbol{s}=d \sigma \boldsymbol{n}$ with $d \sigma$ and $\boldsymbol{n}$ being the volume and the unit outward normal vector of a boundary element of $S(V)$, respectively.

In order to proceed, the whole space is divided into uniform hexahedrons. Simple types of $\mathrm{SE}$ and $\mathrm{CE}$ are defined for each grid point. For example, the conservation element $\mathrm{CE}\left(\mathrm{G}^{\prime}\right)$ is defined by the four-dimensional time-space region $A 1 A 2 A 3 A 4 A 5 A 6 A 7 A 8 A 1^{\prime} A 2^{\prime} A 3^{\prime} A 4^{\prime} A 5^{\prime} A 6^{\prime} A 7^{\prime} A 8^{\prime}$, the solution element $S E\left(G^{\prime}\right)$ constituted by the four hexahedrons ( $B 1 B 3 B 11 B 9 B 1^{\prime \prime} B 3^{\prime \prime} B 11^{\prime \prime} B 9^{\prime \prime}$, $B 2 B 4 B 12 B 10 B 2^{\prime \prime} B 4^{\prime \prime} B 12^{\prime \prime} B 10^{\prime \prime}, \quad B 5 B 6 B 7 B 8 B 5^{\prime \prime}$ $B 6^{\prime \prime} B 7^{\prime \prime} B 8^{\prime \prime}$ and $A 1^{\prime} A 2^{\prime} A 3^{\prime} A 4^{\prime} A 5^{\prime} A 6^{\prime} A 7^{\prime} A 8^{\prime}$ ) intersecting at $G^{\prime}$. Their neighborhood space is demonstrated in Fig. 1.

Suppose that the integral conservation laws are satisfied in each CE and the physical variables in each SE are approximated by first-order Taylor expansions. Note that $\mathrm{CE}\left(\mathrm{G}^{\prime}\right)$ is related to not only $\mathrm{SE}\left(\mathrm{G}^{\prime}\right)$ but also $\mathrm{SE}(\mathrm{A} 1), \mathrm{SE}(\mathrm{A} 2), \mathrm{SE}(\mathrm{A} 3), \mathrm{SE}(\mathrm{A} 4), \mathrm{SE}(\mathrm{A} 5)$, $\mathrm{SE}(\mathrm{A} 6)$, SE(A7) and $\mathrm{SE}(\mathrm{A} 8)$. With the aid of firstorder Taylor expansions in the nine SEs related to
$\mathrm{CE}\left(\mathrm{G}^{\prime}\right)$, Eq. (4) is equivalent to

$$
U_{G^{\prime}}=\frac{1}{8}\left(\bar{U}+\frac{\Delta t}{\Delta x} \bar{E}+\frac{\Delta t}{\Delta y} \bar{F}+\frac{\Delta t}{\Delta z} \bar{G}\right),
$$

with

$$
\begin{aligned}
& \bar{U}=U\left(\frac{\Delta x}{4}, \frac{\Delta y}{4}, \frac{\Delta z}{4}, 0\right)_{A 1}+U\left(-\frac{\Delta x}{4}, \frac{\Delta y}{4}, \frac{\Delta z}{4}, 0\right)_{A 2} \\
& +U\left(-\frac{\Delta x}{4},-\frac{\Delta y}{4}, \frac{\Delta z}{4}, 0\right)_{A 3}+U\left(\frac{\Delta x}{4},-\frac{\Delta y}{4}, \frac{\Delta z}{4}, 0\right)_{A 4} \\
& +U\left(\frac{\Delta x}{4}, \frac{\Delta y}{4},-\frac{\Delta z}{4}, 0\right)_{A 5}+U\left(-\frac{\Delta x}{4}, \frac{\Delta y}{4},-\frac{\Delta z}{4}, 0\right)_{A 6} \\
& +U\left(-\frac{\Delta x}{4},-\frac{\Delta y}{4},-\frac{\Delta z}{4}, 0\right)_{A 7} \\
& +U\left(\frac{\Delta x}{4},-\frac{\Delta y}{4},-\frac{\Delta z}{4}, 0\right)_{A 8},
\end{aligned}
$$

$$
\begin{aligned}
& \bar{E}=E\left(0, \frac{\Delta y}{4}, \frac{\Delta z}{4}, \frac{\Delta t}{4}\right)_{A 1}-E\left(0, \frac{\Delta y}{4}, \frac{\Delta z}{4}, \frac{\Delta t}{4}\right)_{A 2} \\
& -E\left(0,-\frac{\Delta y}{4}, \frac{\Delta z}{4}, \frac{\Delta t}{4}\right)_{A 3}+E\left(0,-\frac{\Delta y}{4}, \frac{\Delta z}{4}, \frac{\Delta t}{4}\right)_{A 4} \\
& +E\left(0, \frac{\Delta y}{4},-\frac{\Delta z}{4}, \frac{\Delta t}{4}\right)_{A 5}-E\left(0, \frac{\Delta y}{4},-\frac{\Delta z}{4}, \frac{\Delta t}{4}\right)_{A 6} \\
& -E\left(0,-\frac{\Delta y}{4},-\frac{\Delta z}{4}, \frac{\Delta t}{4}\right)_{A 7} \\
& +E\left(0,-\frac{\Delta y}{4},-\frac{\Delta z}{4}, \frac{\Delta t}{4}\right)_{A 8}, \\
& \bar{F}=F\left(\frac{\Delta x}{4}, 0, \frac{\Delta z}{4}, \frac{\Delta t}{4}\right)_{A 1}+F\left(-\frac{\Delta x}{4}, 0, \frac{\Delta z}{4}, \frac{\Delta t}{4}\right)_{A 2} \\
& -F\left(-\frac{\Delta x}{4}, 0, \frac{\Delta z}{4}, \frac{\Delta t}{4}\right)_{A 3}-F\left(\frac{\Delta x}{4}, 0, \frac{\Delta z}{4}, \frac{\Delta t}{4}\right)_{A 4} \\
& +F\left(\frac{\Delta x}{4}, 0,-\frac{\Delta z}{4}, \frac{\Delta t}{4}\right)_{A 5}+F\left(-\frac{\Delta x}{4}, 0,-\frac{\Delta z}{4}, \frac{\Delta t}{4}\right)_{A 6} \\
& -F\left(-\frac{\Delta x}{4}, 0,-\frac{\Delta z}{4}, \frac{\Delta t}{4}\right)_{A 7}-F\left(\frac{\Delta x}{4}, 0,-\frac{\Delta z}{4}, \frac{\Delta t}{4}\right)_{A 8}
\end{aligned}
$$

$$
\begin{aligned}
& \bar{G}=G\left(\frac{\Delta x}{4}, \frac{\Delta y}{4}, 0, \frac{\Delta t}{4}\right)_{A 1}+G\left(-\frac{\Delta x}{4}, \frac{\Delta y}{4}, 0, \frac{\Delta t}{4}\right)_{A 2} \\
& +G\left(-\frac{\Delta x}{4},-\frac{\Delta y}{4}, 0, \frac{\Delta t}{4}\right)_{A 3}+G\left(\frac{\Delta x}{4},-\frac{\Delta y}{4}, 0, \frac{\Delta t}{4}\right)_{A 4} \\
& -G\left(\frac{\Delta x}{4}, \frac{\Delta y}{4}, 0, \frac{\Delta t}{4}\right)_{A 5}-G\left(-\frac{\Delta x}{4}, \frac{\Delta y}{4}, 0, \frac{\Delta t}{4}\right)_{A 6} \\
& -G\left(-\frac{\Delta x}{4},-\frac{\Delta y}{4}, 0, \frac{\Delta t}{4}\right)_{A 7}-G\left(\frac{\Delta x}{4},-\frac{\Delta y}{4}, 0, \frac{\Delta t}{4}\right)_{A 8} .
\end{aligned}
$$

Here $X(d x, d y, d z, d t)_{N}$ are the Taylor expansions of $U, E, F$ and $G$ at the reference point $N$, i.e.

$$
\begin{aligned}
X(d x, d y, d z, d t)_{N}= & X_{N}+\left(X_{x}\right)_{N} d x+\left(X_{y}\right)_{N} d y \\
& +\left(X_{z}\right)_{N} d z+\left(X_{t}\right)_{N} d t
\end{aligned}
$$

Substituting Eq. (7) into Eq. (1), we obtain

$$
\left(U_{t}\right)_{N}=-\left(E_{x}\right)_{N}-\left(F_{y}\right)_{N}-\left(G_{z}\right)_{N} .
$$


Equations (7) and (8) imply that the variables needed to be calculated are $U, U_{x}, U_{y}$ and $U_{z}$ at each grid point, because $E, F$ and $G$ are functions of $U$.

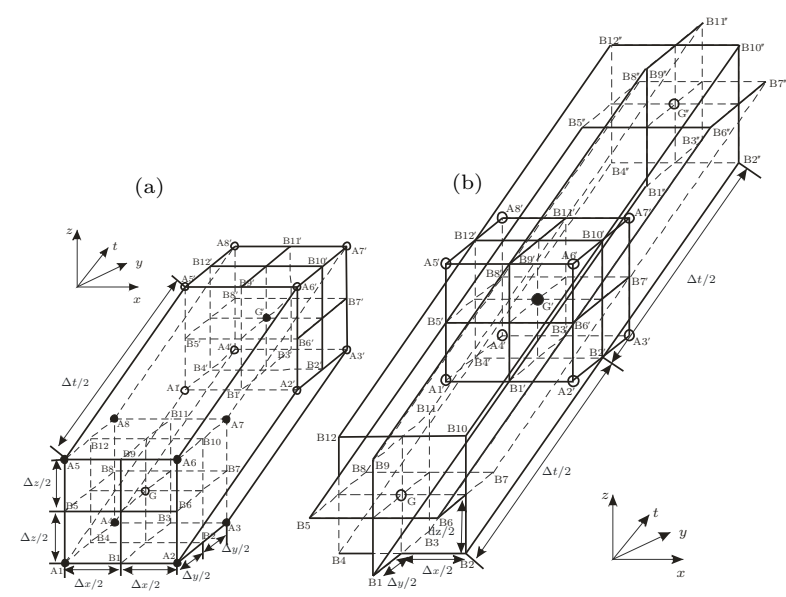

Fig. 1. Definitions of $\mathrm{CE}$ and $\mathrm{SE}$ for the improved threedimensional CE/SE scheme: (a) $\mathrm{CE}\left(\mathrm{G}^{\prime}\right)$, (b) $\mathrm{SE}\left(\mathrm{G}^{\prime}\right)$.

Using the continuous conditions at point $A 1^{\prime}-A 8^{\prime}$, the derivatives of $U$ at $G^{\prime}$ with respect to $x, y$ and $z$ are calculated by

$$
\begin{aligned}
\left(U_{x}\right)_{G^{\prime}} & =W\left[\left(U_{x}\right)_{G^{\prime}}^{-},\left(U_{x}\right)_{G^{\prime}}^{+}, \alpha\right], \\
\left(U_{y}\right)_{G^{\prime}} & =W\left[\left(U_{y}\right)_{G^{\prime}}^{-},\left(U_{y}\right)_{G^{\prime}}^{+}, \alpha\right], \\
\left(U_{z}\right)_{G^{\prime}} & =W\left[\left(U_{z}\right)_{G^{\prime}}^{-},\left(U_{z}\right)_{G^{\prime}}^{+}, \alpha\right],
\end{aligned}
$$

where

$$
\begin{aligned}
& \left(U_{x}\right)_{G^{\prime}}^{-}=-\frac{1}{2 \Delta x}\left[U\left(0,0,0, \frac{\Delta t}{2}\right)_{A 1}+U\left(0,0,0, \frac{\Delta t}{2}\right)_{A 4}\right. \\
& \left.+U\left(0,0,0, \frac{\Delta t}{2}\right)_{A 5}+U\left(0,0,0, \frac{\Delta t}{2}\right)_{A 8}-4 U_{G^{\prime}}\right], \\
& \left(U_{x}\right)_{G^{\prime}}^{+}=+\frac{1}{2 \Delta x}\left[U\left(0,0,0, \frac{\Delta t}{2}\right)_{A 2}+U\left(0,0,0, \frac{\Delta t}{2}\right)_{A 3}\right. \\
& \left.+U\left(0,0,0, \frac{\Delta t}{2}\right)_{A 6}+U\left(0,0,0, \frac{\Delta t}{2}\right)_{A 7}-4 U_{G^{\prime}}\right], \\
& \left(U_{y}\right)_{G^{\prime}}^{-}=-\frac{1}{2 \Delta y}\left[U\left(0,0,0, \frac{\Delta t}{2}\right)_{A 1}+U\left(0,0,0, \frac{\Delta t}{2}\right)_{A 2}\right. \\
& \left.+U\left(0,0,0, \frac{\Delta t}{2}\right)_{A 5}+U\left(0,0,0, \frac{\Delta t}{2}\right)_{A 6}-4 U_{G^{\prime}}\right], \\
& \left(U_{y}\right)_{G^{\prime}}^{+}=+\frac{1}{2 \Delta y}\left[U\left(0,0,0, \frac{\Delta t}{2}\right)_{A 3}+U\left(0,0,0, \frac{\Delta t}{2}\right)_{A 4}\right. \\
& \left.+U\left(0,0,0, \frac{\Delta t}{2}\right)_{A 7}+U\left(0,0,0, \frac{\Delta t}{2}\right)_{A 8}-4 U_{G^{\prime}}\right], \\
& \left(U_{z}\right)_{G^{\prime}}^{-}=-\frac{1}{2 \Delta z}\left[U\left(0,0,0, \frac{\Delta t}{2}\right)_{A 1}+U\left(0,0,0, \frac{\Delta t}{2}\right)_{A 2}\right. \\
& \left.+U\left(0,0,0, \frac{\Delta t}{2}\right)_{A 3}+U\left(0,0,0, \frac{\Delta t}{2}\right)_{A 4}-4 U_{G^{\prime}}\right],
\end{aligned}
$$

$$
\begin{aligned}
& \left(U_{z}\right)_{G^{\prime}}^{+}=+\frac{1}{2 \Delta z}\left[U\left(0,0,0, \frac{\Delta t}{2}\right)_{A 5}+U\left(0,0,0, \frac{\Delta t}{2}\right)_{A 6}\right. \\
& \left.+U\left(0,0,0, \frac{\Delta t}{2}\right)_{A 7}+U\left(0,0,0, \frac{\Delta t}{2}\right)_{A 8}-4 U_{G^{\prime}}\right] .
\end{aligned}
$$

The weighted average function is defined as

$$
W\left[x_{-}, x_{+}, \chi\right]=\frac{\left|x_{+}\right|^{\chi} x_{-}+\left|x_{-}\right|^{\chi} x_{+}}{\left|x_{+}\right|^{\chi}+\left|x_{-}\right|^{\chi}},
$$

where $\chi$ is an adjustable constant and usually equals to $1-2$.

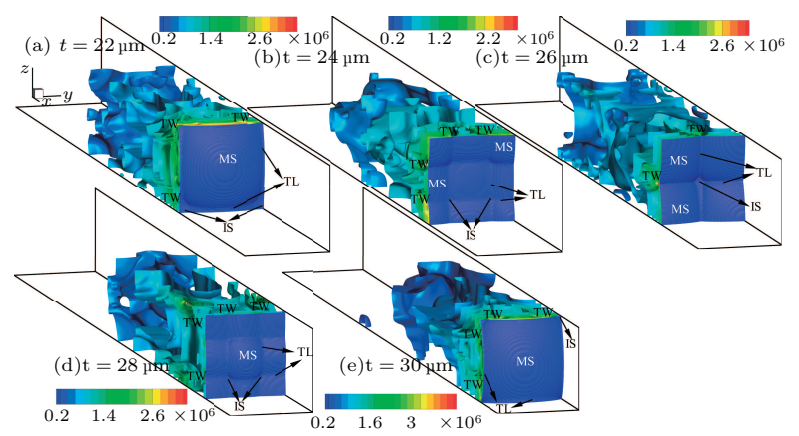

Fig. 2. Pressure space isosurfaces at various times within a period of the rectangular mode.

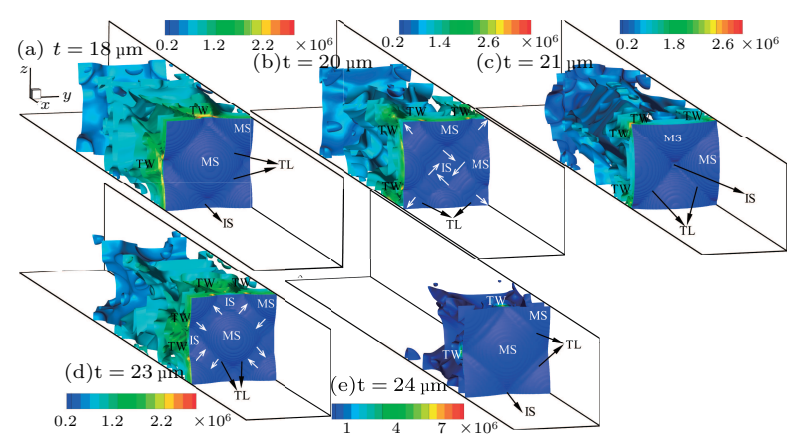

Fig. 3. Pressure space isosurfaces at various times within a period of the diagonal mode.

(a)

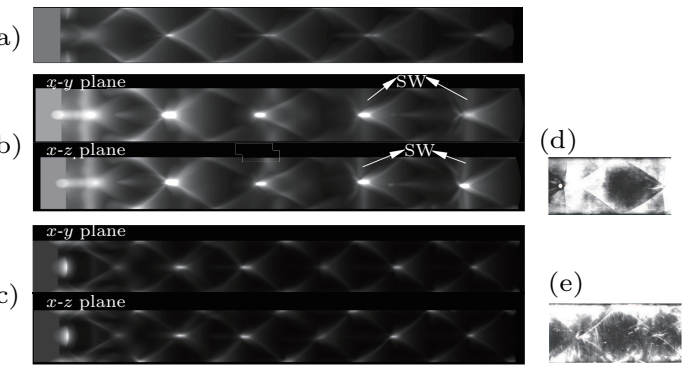

Fig. 4. Comparison of maximum pressure histories. (a) Two-dimensional results; (b) three-dimensional results for rectangular mode; (c) three-dimensional results for diagonal mode, (d) experimental results ${ }^{[16]}$ for rectangular mode; (e) experimental results ${ }^{[16]}$ for diagonal mode.

In the present study, the stoichiometric $\mathrm{H}_{2}-\mathrm{O}_{2}$ cellular detonation propagating in a duct with the size of $8 \times 8 \times 80 \mathrm{~mm}(L / d=10)$ are simulated. All the simulations have the same grid resolution of 
$101 \times 101 \times 1001$. The initial pressure and temperature are $1 \mathrm{~atm}$ and $298 \mathrm{~K}$, respectively. The ignition condition in the channel's left is $40 \mathrm{~atm}$ pressure. The computing parameters of the two-step reaction model for the stoichiometric $\mathrm{H}_{2}-\mathrm{O}_{2}$ gas mixture are given as $a=1.2 \times 10^{8}, b=8 \times 10^{3}$ and $c=0 .{ }^{[15]}$
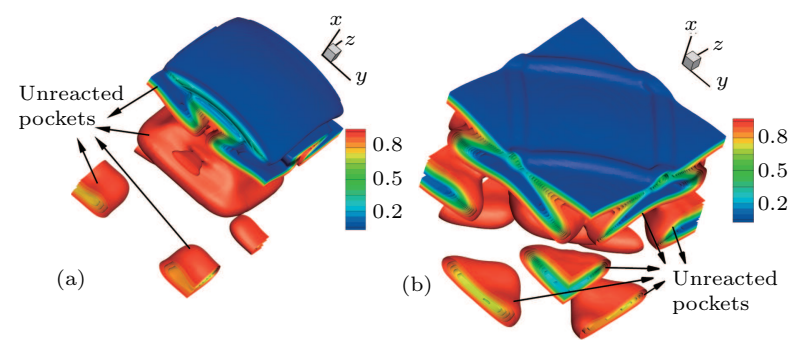

Fig. 5. Space isosurfaces of progress parameter $\beta$ at $30 \mu \mathrm{s}$ : (a) rectangular mode; (b) diagonal mode.

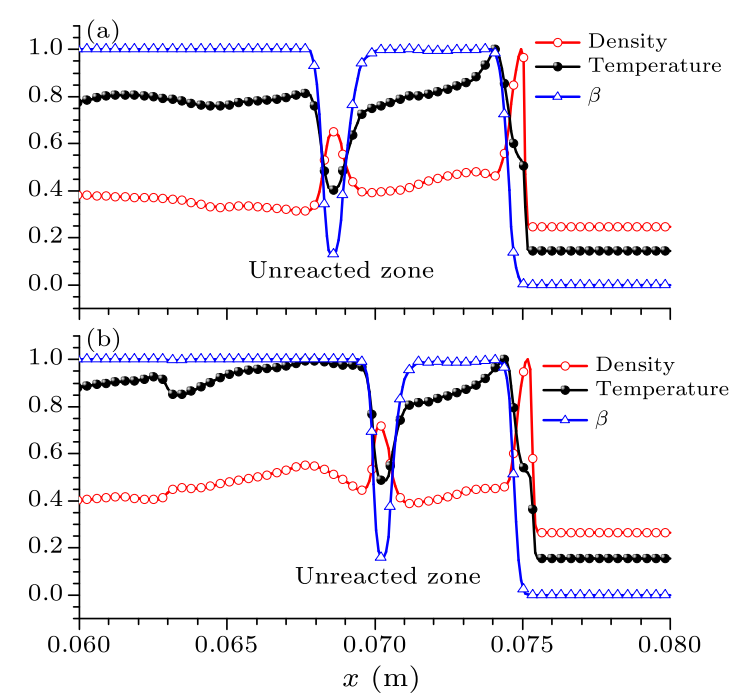

Fig. 6. Local enlargement vision of normalized physical quantities along the center line: (a) rectangular mode, (b) diagonal mode.

Two types of propagation modes, namely a rectangular mode and a diagonal mode, were observed experimentally by Hanana et al. ${ }^{[16]}$ In our simulations, the two types of propagation modes are captured. It seems that the rectangular mode is a more stable mode. The diagonal mode is triggered just under a particular initial condition. Figure 2 shows pressure space isosurfaces at various times within a period of the rectangular mode. The detailed evolution of Mach stems (MS), incident shock (IS), transverse waves (TW) and lines of triple points (TL) is observed intuitively. The rectangular mode consists of two two-dimensional waves which are orthogonal and travel individually with each other. At $24 \mu \mathrm{s}$, two groups of TLs propagate independently, in the vertical and horizontal directions, respectively. Then they collide with each other at the center of the duct at $26 \mu \mathrm{s}$. TWs and high pressure zones where local explosions occur are generated in the vicinity of TLs. An entire cycle is about $8 \mu \mathrm{s}$.
Similarly the results for the diagonal mode are shown in Fig. 3. Unlike the rectangular mode, the TLs move in diagonal directions for this mode. An entire cycle is about $6 \mu \mathrm{s}$, which is about three quarters of that of the rectangular mode. Figure 4 shows the calculated and experimental ${ }^{[16]}$ results comparison of maximum pressure histories. The cellular pattern of rectangular mode is similar to that of the two-dimensional case. However, there exists slapping waves (SW) which are formed by the TLs propagating in the direction perpendicular to the plane. With respect to the diagonal mode, the cell length is about three quarters of that obtained in two-dimensional simulations. There is no SW for this mode. The three-dimensional numerical results agree well with the experimental results.

In Tsuboi's study, ${ }^{2]}$ he formation of unreacted pockets behind the detonation was observed only in the results for rectangular mode in phase. However, in the present study, the formation of unreacted pockets for the two modes mentioned above is captured. The detailed distribution of unreacted pockets is shown by space isosurfaces of $\beta$, which represents the progress parameter of the exothermic reaction. As seen in Fig. 5, there are a large number of unreacted gases $(\beta<1)$ behind the detonation front. It seems that the unreacted pockets have a tendency to be formed near the transverse waves. The unreacted pockets are wrapped up by MS and TW or the tube wall. Figure 6 shows the local enlargement vision of normalized physical quantities along the center line of the duct at $30 \mu \mathrm{s}$. It can be clearly seen that there is an unreacted zone with $\beta<1$. The gases in the zone have lower temperature and higher density compared to the surrounding gases. This is because the gases in the unreacted zone were compressed by MS and TW.

In conclusion, the present scheme is a genuinely three-dimensional scheme. The numerical results show that the complex three-dimensional detonation phenomenon can be simulated accurately by using the improved CE/SE scheme.

\section{References}

[1] Roy G D et al 2004 Prog. Energy Combust. Sci. 30545

[2] Tsuboi N, Katoh S and Hayashi 2002 Prog. Combust. Inst. 292783

[3] Deledicque V and Papalexandris M V 2006 Combust. Flame 144821

[4] Dou H S et al 2008 Combust. Flame 154644

[5] Chang S C 1995 J. Comp. Phys. 119295

[6] Chang S C et al 1999 J. Comput. Phys. 15689

[7] Wang X Y et al 2000 AIAA 2000-0474

[8] Wang G et al 2007 Chin. Phys. Lett. 243563

[9] Wang G et al 2010 Chin. Phys. Lett. 27024701

[10] Wang G et al 2010 Comput. Fluids 39168

[11] Wang G et al 2011 Comput. Phys. Commun. 1821589

[12] Wang J T et al 2009 Comput. Fluids 38544

[13] Chen Q Y et al 2010 J. Comput. Phys. 2297503

[14] Chen Q Y and Liu K X 2011 Chin. Phys. Lett. 28064602

[15] Sichel M et al 2002 Proc. Roy. Soc. Londond A 45849

[16] Hanana M et al 2001 Shock Waves 1177 